\title{
Fiber Optic Strain and Temperature Sensing: Overview of Principles
}

\author{
Rainer Engelbrecht \\ Polymer Optical Fiber Application Center (POF-AC), \\ Technische Hochschule Nürnberg Georg Simon Ohm, \\ Wassertorstraße 10, 90489 Nürnberg, Germany \\ rainer.engelbrecht@th-nuernberg.de
}

\begin{abstract}
:
Fiber-optic sensing of temperature and strain over many advantages over electronic sensors. FiberBragg-Gratings (FBGs) are used for spot sensing, whereas Rayleigh, Brillouin and Raman scattering are used for distributed sensing in long fibers. In this article, these sensor principles are briefly reviewed.
\end{abstract}

Key words: Fiber sensors, fiber Bragg gratings, reflectometry, distributed sensing

\section{Introduction}

Fiber-optic sensors offer distinctive advantages compared to electronic sensors or sensors with electrical connection leads, for example:

- Immune to electromagnetic interference,

- galvanically isolated sensor wiring,

- multiplexing capability for large sensor networks,

- low-loss optical fiber transmission for remote or distributed sensing over large distances without compromising sensor readout,

- small, light-weighted and flexible,

- stability in harsh environments,

- suitable for use in EX-zones.

From the plethora of quantities that can be measured with fiber optics, strain and temperature are amongst the most prominent $[1,2]$. In this article, principles of fiber optic strain and temperature sensing are briefly reviewed.

\section{Optical Fibers for Sensing}

Most optical fibers for sensing are the same as fibers used for telecommunications or other fiber optic applications. Such commercially available fibers have prices in the order of a few $€ / m$ and are in most cases not the most expensive part of a fiber optic sensor system. Optical fibers rely on guiding a light wave in the core of a fiber which has a larger optical refractive index $n$ than the surrounding cladding.

Multimode fibers (Fig. 1a) can be described by geometrical optics where a ray of light is kept in the fiber core by manifold total internal reflections on the core-cladding interface. Many modes with different angles of the rays in respect to the fiber axis can propagate in MMFs. Due to the quite large core diameter coupling of a MMF
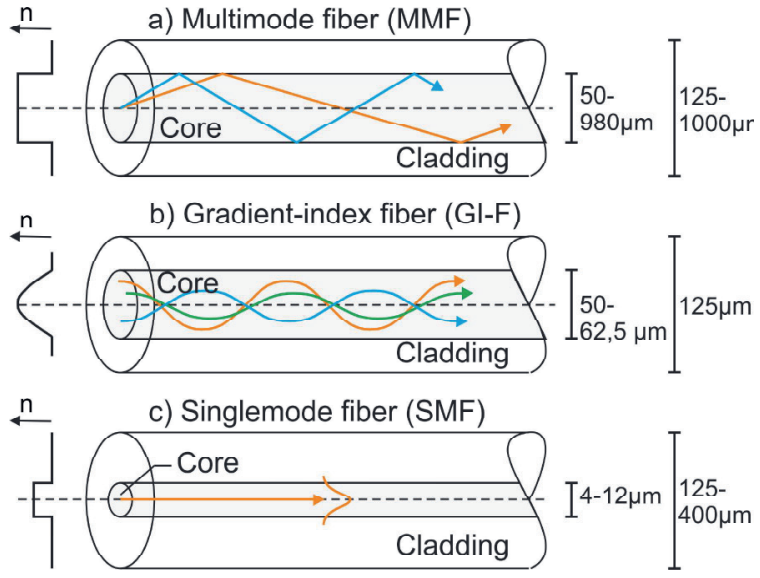

Fig. 1. Commonly used fiber geometries.

to light emitters like LEDs and large detectors is possible, and tolerance to mutual misalignment of fiber connectors is high. However, different modes have differently long propagation paths and thus, propagation times, which leads to temporal dispersion of short light pulses along a MMF. Together with a moderate attenuation (roughly $1 \mathrm{~dB} / \mathrm{km}$ for glass MMFs, wavelength dependent), this limits the use of MMF to short and medium long ranges up to a few $100 \mathrm{~m}$, especially when time-of-flight methods are used for spatially resolved sensing.

Gradient-index (GI) fibers mitigate this mode dispersion by a radially parabolic index profile, providing a higher speed of light to higher-order modes with longer path lengths.

If the core diameter equals only a few optical wavelengths and if the core-cladding index difference is low, light propagation has to be described by electromagnetic wave optics. 
In singlemode fibers, only one spatial electromagnetic field geometry called a mode can propagate. The mode propagates with a well-defined phase velocity which is required for a high spatial resolution of time-of-flight sensors and for fiber Bragg gratings (FBG), usually. Also, SMFs offer the lowest attenuation of all fibers which is $<0,2 \mathrm{~dB} / \mathrm{km}$ at $1550 \mathrm{~nm}$ in the near infrared, making them a perfect match for longrange distributed sensing. However, the small core diameter requires lasers for efficiently coupling of light into the fiber, typically laser diodes (LD).

\section{Materials for optical fibers}

Typically, fused silica glass (amorphous $\mathrm{SiO}_{2}$ ) is used as material for optical fibers and fiber sensors of all types (MMF, GI, SMF). Different dopants like germania $\left(\mathrm{GeO}_{2}\right)$ or fluorine $(\mathrm{F})$ are used to change the refractive index in core or cladding. Fused-silica fibers offer the lowest losses and can sustain temperatures up to $800^{\circ} \mathrm{C}$ in principle, but are often limited to lower temperatures because of a protective polymer coating on top of the cladding. Strain is limited to $1 \%$ or $10000 \mu \varepsilon$ before the probability of a fiber damage gets prohibitive.

Polymer optical fibers (POF) are commonly made of PMMA and are available typically as MMF with large diameters up to $1 \mathrm{~mm}$. Their large diameter and high acceptance angle for light makes coupling to LEDs and connecting fibers easy, they are quite cheap, and withstand very small bending radii and a high strain up to $10 \%$. However, the large attenuation of a PMMA POF in the order of $100 \mathrm{~dB} / \mathrm{km}$, which is lowest in the visible spectral range, limits the application to short range systems, only. GI-POFs made of cyclic fluoropolymers offer lower attenuation and lower mode dispersion at a higher cost.

\section{Time-of-flight POF strain sensors}

Polymer optical fibers (POF) are well suited for the dynamic measurement of relatively large strains on extended structures as POF withstand high relative elongations. Fig. 2 shows and example for a POF sensor for the measurement of path-integrated strain or elongation $\Delta L$ of a structure compared to a reference [3].

The light intensity of a LED or a laser diode is modulated harmonically by an oscillator at a RF frequency $f$. The light intensity is detected by photo diodes and an RF phase comparator measures the phase difference of the modulation. The phase difference $\Delta \Phi$ is directly proportional to the path-integrated strain or elongation of the structure $\Delta L$ :

$\Delta \Phi=2 \pi f \Delta L / c$

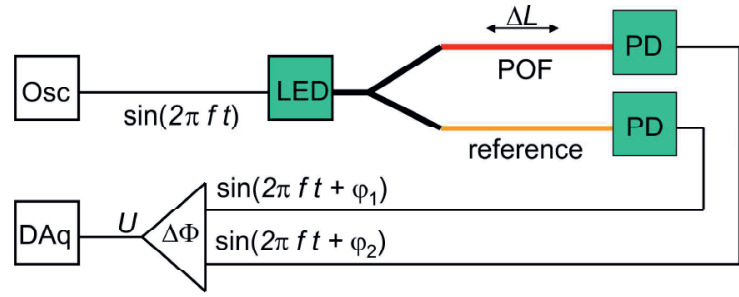

Fig. 2. POF-based sensor for path-integrated strain or elongation of a structure.

where $c$ is the speed of light in the fiber. With a fast data acquisition, dynamic strain or vibrations of several $\mathrm{Hz}$ can be measured. No spatial resolution of the strain along the sensing fiber is provided here, but can be added using i-OFDR as described later [4].

\section{Point-sensing with Fiber Bragg Gratings}

A fiber Bragg grating (FBG) is a fiber with a periodic modulation $n(z)$ of the refractive index in the core of a fiber as shown in Fig. 3 . If the light wavelength matches the Bragg condition

$\lambda_{\mathrm{B}}=2 n_{\mathrm{eff}} \Lambda_{\mathrm{B}}$,

light gets strongly reflected by coherent superposition of many weak partial reflections on the index changes. Here, $\Lambda_{\mathrm{B}}$ is the geometric period of the index grating, and $n_{\mathrm{eff}}$ is the effective refractive index for a particular mode propagating in the core. FBGs are manufactured by side-illumination with intense and patterned laser radiation, which causes a permanent change of the refractive index inside the glass volume. As a specialty, draw-tower gratings are inscribed already during fiber manufacturing [5].

To avoid ambiguity, singlemode fibers (SMF) with only one propagating mode are commonly used, giving rise to one well-defined Bragg wavelength $\lambda_{\mathrm{B}}$. The peak reflectivity can be $>99 \%$, for example for applications as mirrors in fiber lasers. However, reflectivities of a few \% are sufficient for sensing, if a single-peak and narrow-band (few ten $\mathrm{pm}$ ) reflection profile is ensured.

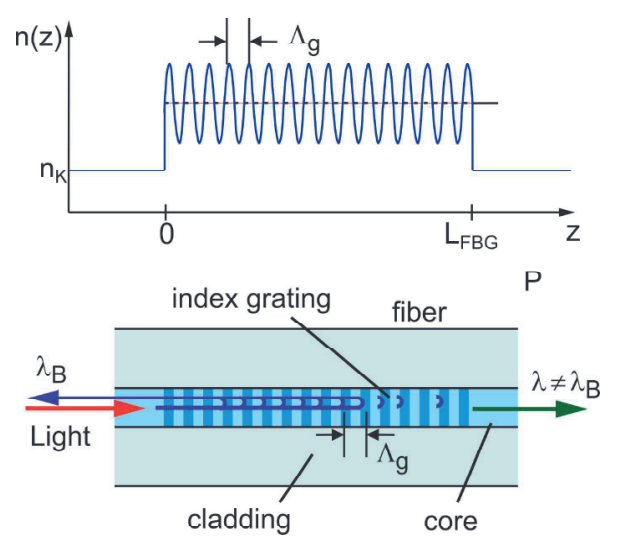

Fig. 3: Principle of a fiber Bragg grating (FBG) 


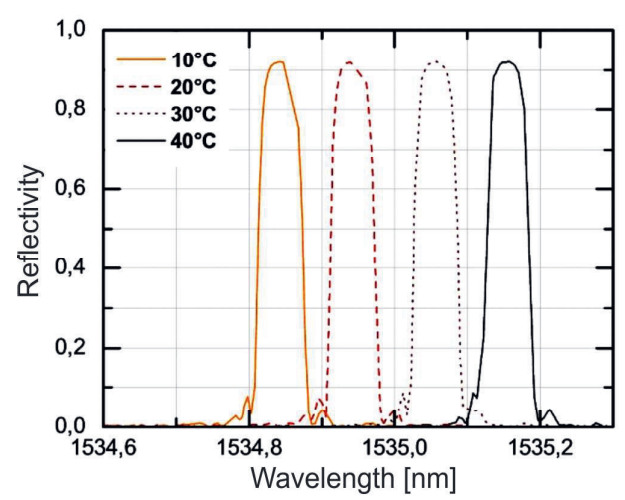

Fig. 4: Temperature shift of FBG reflection spectra

Temperature $T$ and strain $\varepsilon=\Delta L / L$ have impact on both, refractive index $n_{\text {eff }}$ and the geometrical period $\Lambda_{\mathrm{B}}$, and thus, on the Bragg wavelength $\lambda_{B}$. For sensing, the Bragg wavelength $\lambda_{\mathrm{B} 0}$ at a reference temperature $T_{0}$ and at zero strain has to be known. Then, changes in temperature and strain can be monitored by measuring the shift of the Bragg wavelength as shown in Fig. 4. Typical sensitivity coefficients are

$\frac{\Delta \lambda_{\mathrm{B}}}{\lambda_{B 0}}=8 \cdot 10^{-6} \mathrm{~K}^{-1} \Delta T+0,8 \varepsilon$.

One remarkable feature of FBG sensing is the possibility of chaining many FBGs sequentially along one fiber, and multiplexing of these discrete sensing points by different reference wavelengths $\lambda_{\mathrm{B} i}$ as shown in Fig. 5. For readout, a broadband light source, for example a superluminescent diode, is coupled into the sensor fiber. With a spectrometer, the individual shifts of the Bragg wavelengths due to temperature and strain are monitored. Thus, a single FBG interrogator unit can address several tens of distinct sensor points, limited by the spectral bandwidth of the interrogator, only.

Compact spectrometers typically consist of one diffraction grating and a line array of photodetectors to measure the reflection spectrum without moving parts. Such devices are also called a polychromator. Although the spectral resolution of such compact spectrometers is limited to a few $100 \mathrm{pm}$, the peak or centroid of an individual FBG reflection spectrum can be detected with a precision in the order of $10 \mathrm{pm}$ by interpolation, provided that the FBG spectra don't overlap and the signal-to noise ratio (SNR) is sufficiently high. Thus, a precision of temperature and strain readout in the order of $1 \mathrm{~K}$ or $10 \mu \varepsilon$ can be achieved. Another realization of systems for FBG interrogation uses tunable lasers as light source, and simple photo detectors for acquisition of spectra while tuning the light source. Spatial multiplexing of FBGs having the same

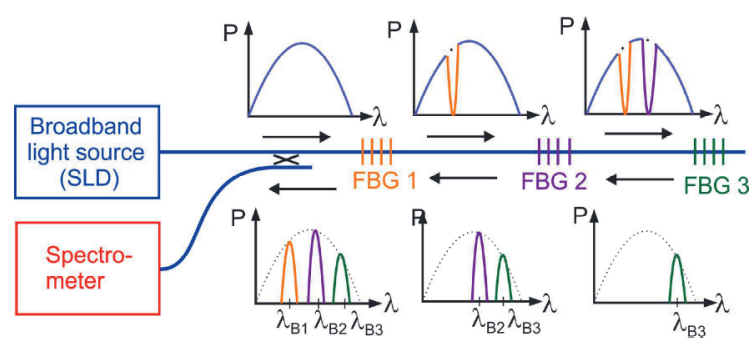

Fig. 5: Wavelength multiplexing of $F B$ chains

wavelength has also been demonstrated using either coherent or incoherent optical frequencydomain reflectometry (OFDR) [6].

One problem of FBG sensing (and other fiber sensors as well) is the simultaneous sensitivity to strain and temperature which gives rise to cross-talk as typically only one quantity should measured. For temperature sensing, the FBG fiber can be installed in loose tubes without influence of strain. For strain sensing the FBG has to be bond to the object under test firmly, and the influence of temperature has to be compensated by an additional temperature sensor, e.g. a second FBG. Combined modeinterference sensors in few-mode fibers with FBGs have demonstrated to measure strain and temperature simultaneously with little cross talk, but at cost of limited multiplexing of many sensors.

FBGs are well-suited fiber-optic sensors at a moderate cost for point-type sensing of up to several tens of sensor spots. As the measured information is coded in the wavelength of the light, fiber attenuation and losses on fiber connectors do not compromise sensor readout, as long as enough light hits the spectrometer.

\section{Distributed Sensing by Light Scattering}

Light is scattered during propagation in a fiber by many intrinsic processes like Rayleigh, Brillouin and Raman scattering. With all of these processes, sensing of physical quantities like temperature or strain (the later not with Raman) is possible as described in the next sections. Unlike FBG sensing, no special preparation of the fiber is required, the bare fiber itself acts as sensor element. However, there are no discrete sensor points that can be addressed by wavelength multiplexing, for example. Instead, a time-of-flight principle has to be used, which assigns any location $z$ along a fiber a unique time $t$ that is required for a light pulse to travel from a light source to this point and backwards. Thus, a continuous sensing of quantities with virtually 1000 s of sensor spots along a fiber is possible, referred to as distributed sensing [1]. 


\section{Spatially resolved backscatter measurement}

The most simple principle to achieve a spatial resolution of backscatter measurements along a fiber is well-known as optical time-domain reflectometry (OTDR). A short light pulse is launched into the fiber, and the reflected power $P(t)$ is recorded over time. By the speed of light $c=c_{0} / n_{\text {eff }}$ of a mode in the fiber, time is proportional to the location of a reflection event:

$z=t \cdot c / 2$.

By proper calibration, the reflection profile $R(z)$ can be calculated.

The resolution $\Delta z$ of two point-like reflections or spatial resolution for distributed sensing is given by the duration of the light pulse $\Delta t$ :

$\Delta z=\Delta t \cdot c / 2$.

For a typical pulse width of $1 \mathrm{~ns}$ and $n_{\mathrm{eff}} \approx 1,5$, a spatial resolution of $10 \mathrm{~cm}$ is achieved in singlemode fibers. In multimode fibers, spatial resolution is deteriorated by pulse dispersion over longer fiber lengths.

With this technology, spot or point-type reflections from fiber end facets or from FBGs can be measured as well as distributed scattering like Rayleigh, Raman or Brillouin, in principle. The detection range is limited by fiber attenuation, which generates an exponential decay of optical power along the fiber.

One disadvantage of OTDR is the low average optical power in the fiber as only one short pulse can propagate at a time to avoid ambiguity. This leads to a low SNR which requires averaging over many pulse cycles, unless optical pulses with very high peak power are employed.

This problem is mitigated by optical frequencydomain reflectometry (OFDR) where a continuous wave (cw) laser is used. In incoherent OFDR (i-OFDR), the intensity of the laser is then modulated harmonically with different RF frequencies $f$ covering an electrical bandwidth of $B$. Then the reflected power $P(f)$ for each RF modulation frequency is measured, resembling a transfer function in the frequency domain. The inverse Fourier transform then yields the impulse response in the time domain which is equal to the OTDR result $P(t)$. As the average optical power is typically higher using OFDR, a better SNR is achieved in less time compared to OTDR.

Spatial resolution is inverse to the bandwidth of $\mathrm{RF}$ frequencies used:

$\Delta z=c /(2 B)$,

so high-speed opto-electronical components having a large RF bandwidth are required for

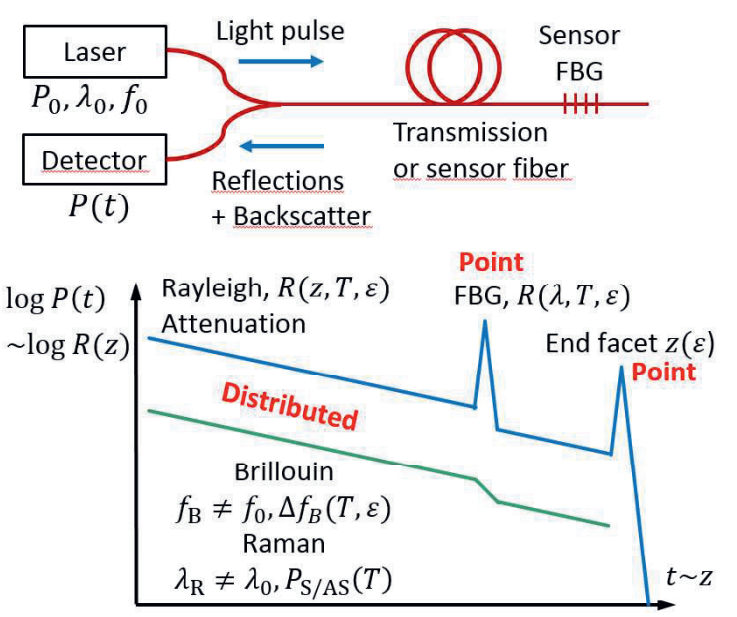

Fig. 7: Optical time-domain reflectometry (OTDR) for point reflections and distributed scattering.

modulation and detection. In principle, this is also true for OTDR when using short pulses. For example, $B=1 \mathrm{GHz}$ yields $\Delta z=10 \mathrm{~cm}$. With both, OTDR and OFDR, fiber lengths of some $10 \mathrm{~km}$ can be monitored using low-loss SMFs.

For coherent OFDR (c-OFDR), a true cw laser tunable in wavelength or optical frequency, respectively, is used in an Michelson interferometric setup. During tuning, an interferogram is recorded by superposition of light from a reference path and from the backscatter in the fiber. The inverse Fourier transform of these interferogram yields the impulse response or spatial reflection profile $R(z)$ as well. For c-OFDR Eq. (6) still holds, but with very large optical tuning bandwidths $B$ of the laser in the order of several $10 \mathrm{~nm}$ or several $\mathrm{THz}$, respectively. Thus, an excellent spatial resolution of a few $10 \mu \mathrm{m}$ is achieved, at the cost of a precise and costly tunable laser source. The maximum range is limited to some $100 \mathrm{~m}$ because of coherence properties of the laser and the bandwidth of the electronics for recording a fast-oscillating interferogram for distant reflections.

Finally, code-division optical reflectometry (CDOR) uses long and noise-like pseudorandom digital bit patterns for modulation of the laser intensity. These codes have a very narrow autocorrelation peak in the time domain. For CDOR, the reflected light intensity is acquired and mathematically cross-correlated to a reference by digital signal processing. A narrow correlation peak is generated for each point of reflection along the fiber. For CDOR, no RF components like oscillators or mixers are required, but high-speed digital-analog and analog-digital converters (DAC and ADC) with good bit resolution are used. Again, Eq. (6) holds for spatial resolution at given bandwidth $B$. 


\section{Rayleigh scattering}

Rayleigh scattering results from micro- and mesoscopic fluctuations of the refractive index of the fiber glass, frozen-in from the melt of fiber drawing. For a standard telecom SMF, Rayleigh scattering is the strongest linear scattering process with a typical power ratio of incident to backscattered light per unit length of $-73 \mathrm{~dB} / \mathrm{m}$ at $1550 \mathrm{~nm}$. Clearly, such small reflected powers require efficient detection techniques with a good SNR, as discussed earlier.

The power of Rayleigh scattering is almost independent of temperature and strain. However, if Rayleigh scattering is measured with very high spatial resolution by c-OFDR, a unique spatial reflection profile is obtained for each fiber section, called a fingerprint. Once a complete fingerprint along a singlemode fiber is acquired as a reference $R_{x}(z)$, a differential shift $\Delta z$ of the pattern to $R_{y}(z)$ because of temperature or strain can be detected precisely, using a mathematical cross-correlation [7]. Cross-talk of strain and temperature has to be considered, as discussed at FBGs (which could also be interrogated by cOFDR, actually). Rayleigh c-OFDR systems offer the best spatial resolution and good measurement speed, limited to a few $100 \mathrm{~m}$ of fiber, at a relatively high system cost because of the very precisely tunable laser.

\section{Brillouin Scattering}

Brillouin scattering is caused by scattering of light on acoustic waves, because acoustic waves in matter are density waves which modulate the refractive index. Due to the reflection on an index grating travelling at the speed of sound $v_{\mathrm{A}}$, the light wave with wavelength $\lambda_{0}$ gets a specific Doppler shift of its optical frequency:

$\Delta f_{\mathrm{B}}=2 n_{\mathrm{eff}} v_{\mathrm{A}} / \lambda_{0}$.

For a wavelength of $1550 \mathrm{~nm}$, this Brillouin frequency shift is $11 \mathrm{GHz}$ in a typical fused-silica singlemode fiber [8]. By heterodyne mixing of the source light of a laser with the backscattered light from the fiber on a high-speed photodetector, the Brillouin frequency $\Delta f_{\mathrm{B}}$ in the photo current can be measured with microwave electronics. Singlemode fibers are preferred to have a well-defined mode index $n_{\text {eff }}$ and thus, Brillouin shift.

Both, strain and temperature change this Brillouin frequency $\Delta f_{\mathrm{B}}$ which is the essence of Brillouin sensing, as shown in Fig. 8 for strain or tensile forces. The relative sensitivity to temperature compared to strain is twice as much as the same ratio for FBGs, but cross-talk has to be considered, too.

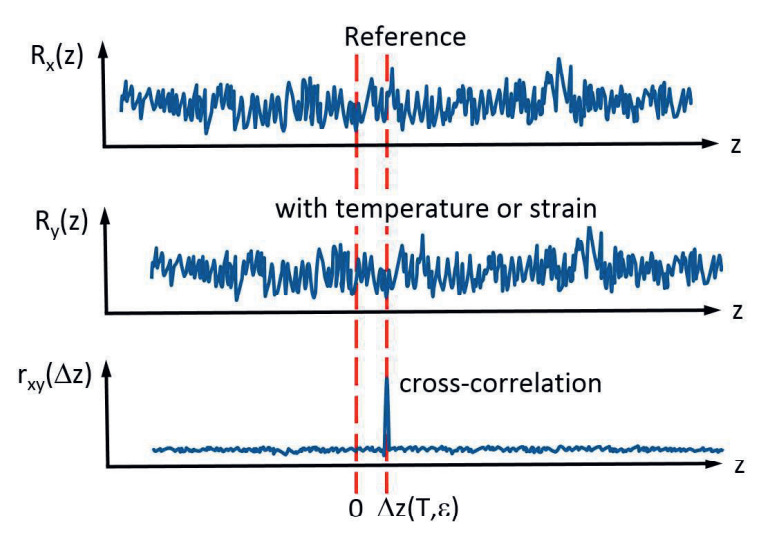

Fig. 8: Fingerprint Rayleigh backscatter profiles can be used to measure temperature or strain.

Brillouin reflectometers rely on spontaneous Brillouin scattering which is caused by thermally excited random acoustic waves in matter. In a standard SMF at $1550 \mathrm{~nm}$, the relative backscatter per unit length is only $-93 \mathrm{~dB} / \mathrm{m}$, two orders of magnitude lower than Rayleigh scattering. Sensitive electronics and averaging are required to obtain useful sensor data.

Brillouin analyzers utilize stimulated Brillouin scattering (SBS) which occurs for two counterpropagating light waves having a frequency difference exactly equal to the Brillouin frequency shift $\Delta f_{\mathrm{B}}$. By a complex interplay of effects like interference, electrostriction and Doppler shift, a significant amplification of one of the light waves is obtained. By measuring this Brillouin gain at different frequency shifts of the counter-propagating light waves, $\Delta f_{\mathrm{B}}$ can be determined with less noise and higher speed [9]. This comes at cost, as both fiber ends have to be optically accessible to the measurement device, in contrast to reflectometers which require only one end of the fiber connected. SMFs are required for SBS because of their small mode diameter and thus, large light intensity to observe nonlinear optical effects.

For spatial resolution time-of-flight methods like OTDR, or preferably i-OFDR and CDOR are used. Because of the low loss in singlemode fibers, distributed Brillouin sensing is possible for very long lengths of many $10 \mathrm{~km}$.

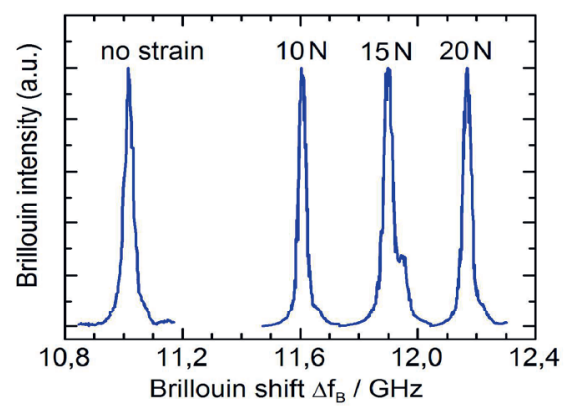

Fig. 9: Brillouin spectra and frequency shift for a SMF with different pulling forces. 


\section{Raman Scattering}

Raman scattering is the scattering of light on the vibrating molecular bonds of matter. In fusedsilica, these bonds vibrate at a wide band of frequencies around $f_{\mathrm{V}} \approx 10 \mathrm{THz}$. This generates two spectral wings in the Raman scattered light (Fig. 10). The wing shifted to lower frequencies in respect to the light source, i.e. shifted to larger wavelengths, is called Stokes scattering. The wing at higher frequencies is Anti-Stokes scattering. Temperature sensing evaluates the power ratio between Stokes $P_{\mathrm{S}}$ and Anti-Stokes $P_{\mathrm{AS}}$ for spontaneous Raman scattering, which is temperature-depended because of thermodynamic and quantum-electronic reasons:

$\frac{P_{\mathrm{AS}}}{P_{\mathrm{S}}} \sim \exp \left\{-\frac{h f_{\mathrm{v}}}{k T}\right\}$,

where $h$ is Planck's and $k$ is Boltzmann's constant [9]. Therefore, the backscattered light is spectrally filtered into a Stokes and Anti-Stokes band, and the power in each band is measured.

Spontaneous Raman scattering is the weakest of all scattering process discussed here, requiring the longest measurement times, in coarse speaking. Stimulated Raman scattering, which would be a much stronger effect, is generally not temperature-dependent, and can not be used for sensing.

Again, OTDR, i-OFDR or CDOR is used for spatial resolution in Raman distributed sensing [10]. MMF or GI fibers are used for lengths up to many $100 \mathrm{~m}$, and SMF for longer lengths of several $\mathrm{km}$ because of lower attenuation and pulse dispersion.

Raman distributed temperature sensing (DTS) has the unique property that it is not influenced by strain. This is more a benefit than a limitation as it avoids cross-talk of strain to DTS.

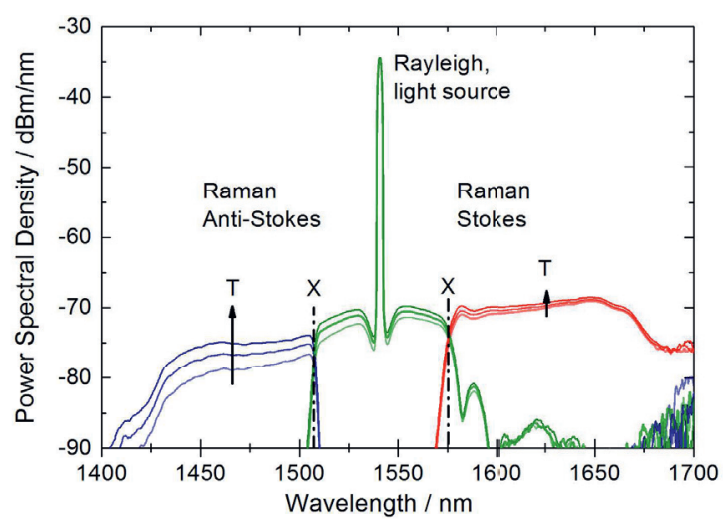

Fig. 10: Example for a fiber backscatter spectrum showing temperature-dependent Raman Stokes and Anti-Stokes scattering and Rayleigh scattering. The $X$ marks the cross-over for spectral filtering of Stokes/Anti-Stokes bands for a Raman DTS.

\section{Conclusion and Outlook}

Fiber optic temperature and strain sensing is widely employed in monitoring of industrial machines and processes, for structural health monitoring, for surveillance systems and many more beyond the scope of this small article. Fiber Bragg gratings offer many sensing spots at moderate cost, whereas high-end distributed sensing with Rayleigh, Brillouin or Raman scattering offer virtually thousands of measurement locations in very long fibers.

Combinations of optical sensing principles offer new applications with added benefits and reduced drawbacks. New fiber types, more costeconomic lasers or other light-sources as well as spectrometers and detectors drive future trends in fiber sensing. Digital signal processing and high-speed high-resolution AD/DA conversion will have the same impact on optical sensing as on optical communications.

The outlook on fiber optic sensing is bright.

\section{References}

[1] X. Bao, L. Chen: Recent progress in Distributed Fiber Optic Sensing. Sensors 12, 8601-8639 (2012).

[2] M. Majumder et al.: Fibre Bragg gratings in structural health monitoring-Present status and applications. Sensors and Actuators, A: Physical 147, 150-164 (2008)

[3] J. Gomez et. al.: Comparing polymer optical fiber, fiber Bragg grating, and traditional strain gauge for aircraft structural health monitoring. Applied Optics 48, 1436-143 (2009)

[4] S. Liehr et al.: Incoherent optical frequency domain reflectometry and distributed strain detection in polymer optical fibers. Meas. Sci. and Technol. 21, 017001 (2010).

[5] M. Rothhardt et al.: High mechanical strength single-pulse draw tower gratings. Proc. SPIE 5579-1, 127-135 (2004).

[6] S. Werzinger et al. Quasi-Distributed Fiber Bragg Grating Sensing Using Stepped Incoherent Optical Frequency Domain Reflectometry. J. Lightwave Technol. 34, 5270-5277 (2016).

[7] A. K. Sang et al.: One centimeter spatial resolution temperature measurements in a nuclear reactor using Rayleigh scatter in optical fiber. IEEE Sensors J. 8, 1375-1380 (2008).

[8] R. Engelbrecht: Nichtlineare Faseroptik. Springer Vieweg Berlin Heidelberg (2014).

[9] S.M. Foaleng et al.: High spatial and spectral resolution long-range sensing using Brillouin echoes. J. Lightwave Technol. 28, 2993-3003 (2010).

[10] E. Karamehmedovic et al.: Fiber-optic distributed temperature sensor using Incoherent Optical Frequency Domain Reflectometry. Proc. SPIE 5363, 107-115 (2004). 